“ (C) 2016 IEEE. Personal use of this material is permitted. Permission from IEEE must be obtained for all other uses, in any current or future media, including

reprinting/republishing this material for advertising or promotional purposes, creating new collective works, for resale or redistribution to servers or lists, or reuse of any copyrighted component of this work in other works." 


\title{
Distributed Condition Monitoring of Renewable Microgrids Using Adaptive-then-Combine Algorithm
}

\author{
Md Masud Rana, Li Li and Steven W. Su \\ Faculty of Engineering and Information Technology \\ University of Technology Sydney, Broadway, NSW 2007, Australia \\ Email:11766084@student.uts.edu.au,Li.Li@uts.edu.au and Steven.Su@uts.edu.au
}

\begin{abstract}
This paper explores the problem of distributed state estimation including packet losses for the environment-friendly renewable microgrid incorporating electricity generating circuits. The problem is becoming critical due to the global warming, increasing green house gas emissions, and practical infeasibility with computational burden of the large-scale centralized power system monitoring. To address the impending problem, a novel distributed microgrid state estimation algorithm is derived in the context of microgrids. Specifically, after modelling the microgrid, this paper proposes a local microgrid state estimation algorithm considering packet losses. Then a novel optimal weighting factor calculation method for the global state estimation is proposed. Particularly, it can automatically adjust the optimal weighting factors for different sensor measurements based on the observation quality, improving the estimation accuracy of the global estimation. Simulations show that the desired state estimation accuracy is achievable.
\end{abstract}

Keywords-Electricity generating circuits, distributed state estimation, Kalman filter, packet losses, smart grid.

\section{INTRODUCTION}

Today the most imperative problems all over the world are energy crisis and global warming, which is vulnerable to the natural disasters [1], [2], [3]. These impending issues are mainly due to the fact that the greenhouse gas emissions from the generation of electricity from the traditional coal and oil based power plants [4]. Fortunately, to mitigate the aforementioned inconveniences at an acceptable level, one of the effective solutions is to use microgrid incorporating decentralized renewable distributed energy resources (DERs) for achieving a wiser and sustainable future. Generally speaking, the power generation pattern of the microgrid incorporating electricity generating circuits depends on the weather and surrounding ambient conditions [5]. Consequently, the microgrid state estimation is needed to estimate the system state in a distributed way, so that it can operate in a normal and secure manner [6]. The centralized state estimator is infeasible due to the communication burdens. To achieve the reliability, minimize energy losses and diversification of energy sources, the distributed microgrid state estimation is one of the prosing research topics in academia, environmentalists and utility operators. Therefore, a communication network is clearly required for transferring information between the microgrid and the estimator at the energy management system (EMS).

Inevitably, the packet losses occur in the transmission of sensor measurements from the microgrid to the smart EMS. There has been a significant research work undertaken to the network control system to investigate the effect of packet losses [7], [8], [9]. For instance, a stochastic event-triggered sensor schedule for the centralized state estimation with packet dropouts is proposed in [10]. Next, a linear coding based centralized minimum mean square estimator with packet losses is presented for sensor networks [11]. A Kalman filter (KF) based linear quadratic Gaussian control for the power system state estimation (centralized) is suggested in [12]. Beside, a decentralized dynamic power system state estimation without packet losses framework is proposed in [7], [13], [14]. Driven by the aforementioned motivations, a novel distributed microgrid state estimation algorithm is derived in this paper under packet loss conditions. The specific technical contributions of the paper are summarized as follows:

- First of all, the environment-friendly renewable microgrid is modelled in a distributed way. In other words, the microgrid is represented as a discrete-time linear state-space equation which is easy to implement in real-time situations. Then the smart sensors are deployed to obtain the microgrid state information at the smart energy management unit.

- This paper proposes an adaptive-then-combine diffusion Kalman filtering algorithm under packet losses condition. The adaptive algorithm is obtained by minimizing the estimation error covariance matrix of the local estimators.

- This paper proposes an optimal weighting factor calculation method for the global distributed state estimation. It can automatically adjust the optimal weighting factors for different sensor measurements based on the observation quality, reducing the impact of impairments on the global state estimation.

\section{MODELliNG A RENEWABLE MICROGRID}

Figure 1 shows a typical DC microgrid structure incorporating electricity generating circuits [15], [16]. Three different type of local loads such as Constant Power Loads (CPL), Constant Current Loads (CCL) and Constant Resistive Loads (CRL) are connected to the microgrid.

\section{A. Mathematical dynamic model of power sources}

In this work, solar photovoltaic arrays can be regarded as a voltage source $E_{p}$ in series with an internal resistance $R_{p}$ [15], [17]. Here, $f_{p}$ denotes the ratio between output current and input current of DC/DC converter. Based on Kirchhoff's 


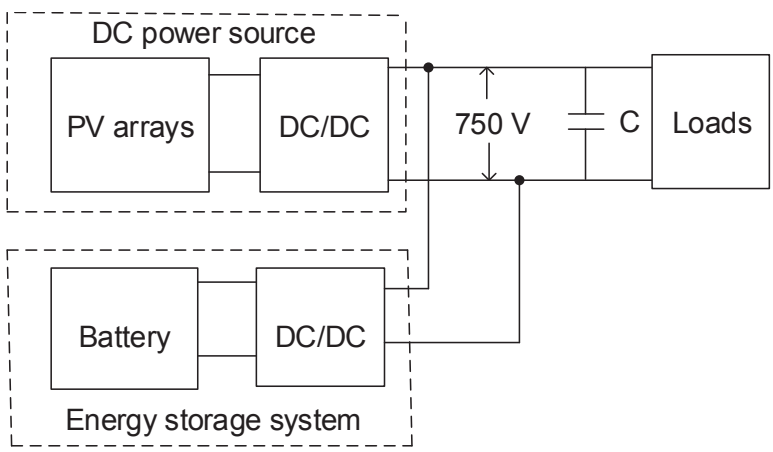

Fig. 1: DC microgrid with multiple types of loads [15].

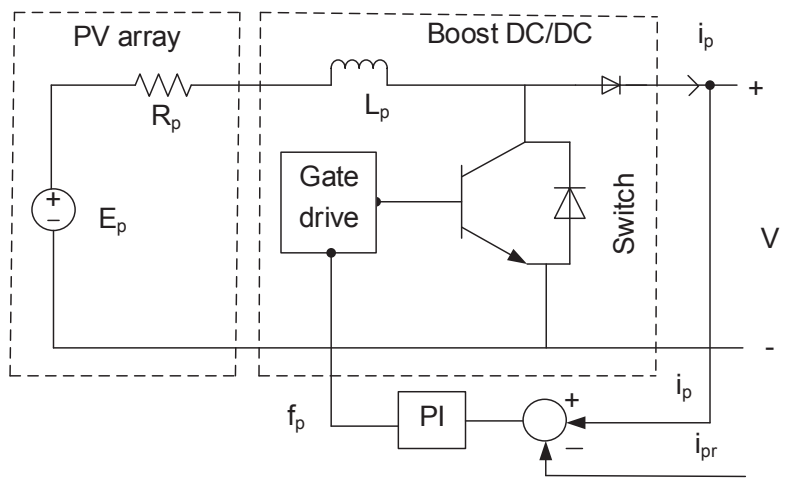

Fig. 2: Model of DC power source with PV array [15].

current law, the equations of the DC power source can be expressed as:

$$
\begin{aligned}
& \dot{i}_{p}=-\left(R_{p} i_{p}-f_{p} E_{p}+f_{p}^{2} V\right) / L_{p}, \\
& f_{p}=f_{p P}+f_{p I}, \\
& f_{p P}=P_{p P}\left(i_{p}-i_{p r}\right), \\
& \dot{f}_{p I}=P_{p I}\left(i_{p}-i_{p r}\right),
\end{aligned}
$$

where $i_{p}$ is the output current of power source, $i_{p r}$ is the reference current of power source, $L_{p}$ is the inductance of PV converter, $V$ is the DC bus voltage, $f_{p P}$ is the proportion part of current ratio $f_{p}, f_{p I}$ is the integral part of current ratio $f_{p}, P_{p P}$ and $P_{p I}$ are the proportional and integral coefficient of proportional and integral controller, respectively [15].

\section{B. Mathematical dynamic model of energy storage systems}

In the energy storage model, the battery is modelled by a series of an equivalent capacitor $C_{b}$ and an internal resistance $R_{b}$. The expression of the capacitor is:

$$
C_{b}=\frac{C_{c a p}}{V_{u p}-V_{l o}}
$$

where $C_{c a p}$ is the battery capacity, $V_{u p}$ and $V_{l o}$ are the upper and lower limit of battery voltage, respectively. The energy

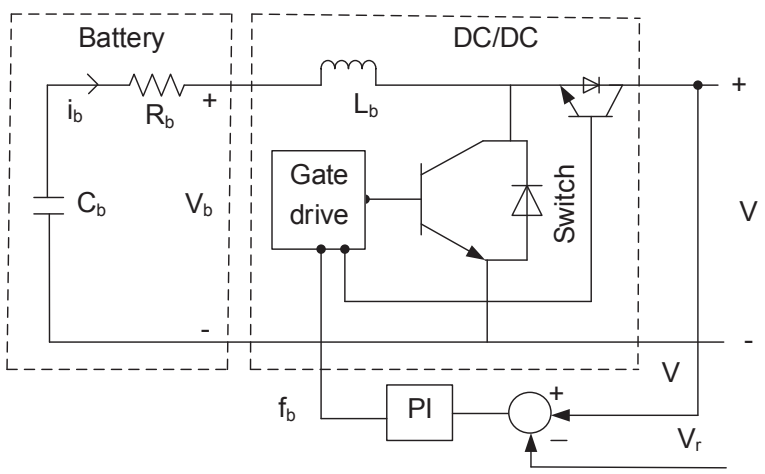

Fig. 3: Model of battery energy storage system [15].

storage system is expressed as follows:

$$
\begin{aligned}
& \dot{i}_{b}=-\left(R_{b} i_{b}-f_{b} V_{b}+f_{b}^{2} V\right) / L_{b}, \\
& f_{b}=f_{b P}+f_{b I}, \\
& f_{b P}=P_{b P}\left(V-V_{r}\right), \\
& \dot{f}_{b I}=P_{b I}\left(V-V_{r}\right), \\
& \dot{V}=-\frac{i_{b}}{C_{b} f_{b}}
\end{aligned}
$$

where $i_{b}$ is the battery output current, $f_{b}$ denotes the ratio between battery output current and battery input current, $V_{b}$ is the battery voltage, $L_{b}$ is the battery inductance, $V_{r}$ is the reference bus voltage, $f_{b P}$ is the proportion part of current ratio $f_{b}, f_{b I}$ is the integral part of current ratio $f_{b}, P_{b P}$ and $P_{b I}$ are the proportional and integral coefficient of proportional and integral controller, respectively [15].

\section{Mathematical dynamic model of loads}

The relationship between CRL load current and voltage can be expressed as:

$$
i_{C R L}=\left(\frac{P_{C R L}}{V_{\text {rat }}^{2}}\right) v,
$$

where $i_{C R L}$ is the current of CRL, $P_{C R L}$ is the load capacity, $V_{\text {rat }}$ is the rated voltage and $v$ is the voltage of CRL. The relationship between CCL load current and voltage can be expressed as:

$$
i_{C C L}=\left(\frac{P_{C C L}}{V_{\text {rat }}}\right),
$$

where $i_{C C L}$ is the current of CRL, $P_{C C L}$ is the load capacity and $V_{\text {rat }}$ is the rated voltage. Finally, the relationship between CPL load current and voltage can be expressed as:

$$
i_{C P L}=\frac{P_{C P L}}{v},
$$

where $i_{C P L}$ is the current of CPL, $P_{C P L}$ is the load capacity and $v$ is the voltage of CPL. So, the function between load current and voltage can be expressed as follows:

$$
i_{\text {load }}=\left(\frac{P_{C R L}}{V_{\text {rat }}^{2}}\right) v+\left(\frac{P_{C C L}}{V_{\text {rat }}}\right)+\frac{P_{C P L}}{v} .
$$


The current relation in Fig. 1 can be expressed as follows [15]:

$$
\dot{V}=\frac{i_{p}+i_{b}-i_{\text {load }}}{C},
$$

where $C$ is the equivalent capacitance between transmission lines as shown in Fig. 1.

\section{Linearization and discretization of microgrid model}

The linearized microgrid model described by (1), (3) and (8) can be expressed as follows [15]:

$$
\dot{\mathbf{x}}=\mathbf{A x}+\mathbf{B u}+\mathbf{n},
$$

where $\mathbf{A}$ is the state matrix, $\mathbf{x}=$ $\left[\begin{array}{llllll}\Delta i_{p} & \Delta f_{p I} & \Delta i_{b} & \Delta f_{b I} & \Delta V_{b} & \Delta V\end{array}\right]^{\prime}$ is the state deviation from the working reference point $\mathbf{x}_{r}, \mathbf{B}$ is the input matrix, $\mathbf{u}=\left[\Delta E_{p} \Delta R_{p} \Delta R_{b}\right]$ is the input signal deviation, and $\mathbf{n}$ is the zero mean process noise whose covariance matrix is $\mathbf{Q}_{n}$. The coefficient matrices $\mathbf{A}$ and $\mathbf{B}$ can be represented by: $\mathbf{A}=\left[\begin{array}{cccccc}-A_{11} & -A_{12} & 0 & 0 & 0 & -\frac{f_{p r}^{2}}{L_{p}} \\ P_{p I} & 0 & 0 & 0 & 0 & 0 \\ 0 & 0 & -\frac{R_{b}}{L_{b}} & -A_{34} & -f_{b r} & -A_{36} \\ 0 & 0 & 0 & 0 & 0 & P_{b I} \\ 0 & 0 & -\frac{1}{C_{b} f_{b r}} & \frac{i_{b r}}{C_{b} f_{b r}^{2}} & 0 & \frac{i_{b r}}{C_{b} f_{b r}^{2}} P_{b P} \\ \mathbf{B} & =\left[\begin{array}{ccc}-f_{p r} & i_{p r} & 0 \\ 0 & 0 & 0 \\ 0 & 0 & i_{b r} \\ 0 & 0 & 0 \\ 0 & 0 & 0 \\ 0 & 0 & 0\end{array}\right], \text { where } A_{11}=\frac{2 f_{p r} V_{r} P_{p P}+R_{p}-P_{p P} E_{p}}{L_{p}},\end{array}\right.$, $A_{12}=\frac{2 f_{p r} V_{r}-E_{p}}{L_{p}}, \quad A_{36}=\frac{2 f_{b r} V_{r} P_{b P}-V_{b r} P_{b P}+f_{b r}^{2}}{L_{b}}$, $A_{34}=\frac{2 f_{b r V_{r}-V_{b r}}}{L_{b}}$, and $A_{66}=\frac{1}{C}\left(\frac{P_{l o a d}}{V^{2}}-\frac{1}{R_{\text {load }}}\right)$. In order to apply the discrete version of the distributed state estimation method, the discretisation of the state-space model is used. Based on the standard Euler formula, (9) can be transformed into the following form:

$$
\mathbf{x}_{k+1}=\mathbf{A}_{d} \mathbf{x}_{k}+\mathbf{B}_{d} \mathbf{u}_{k}+\mathbf{n}_{k},
$$

where $\mathbf{A}_{d}=\mathbf{I}+\mathbf{A} \Delta t, \Delta t$ is the discretization step size, $\mathbf{B}_{d}=$ $\mathbf{B} \Delta t$, and $\mathbf{n}_{d}(k)=\Delta t \mathbf{n}(k)$ with the covariance matrix $\mathbf{Q}$.

\section{DISTRIBUTED SYSTEM MODEL}

The proposed distributed system model including packet losses is described in Fig. 4. In order to simplify our discussion, here, it is assumed there are $\mathrm{N}=4$ observation stations in the distribution power network. The measurements from the system states are described by a set of sensors as follows:

$$
\mathbf{z}_{k}^{i}=\mathbf{C}^{i} \mathbf{x}_{k}+\mathbf{w}_{k}^{i}
$$

where $\mathbf{z}_{k}^{i}$ is the observation information by the $\mathrm{i}$-th estimator at the time instant $\mathrm{k}, \mathbf{C}^{i}$ is the observation matrix, and $\mathbf{w}_{k}^{i}$ is the zero mean measurement noise whose covariance matrix is $\mathbf{R}_{k}^{i}$. Taking into account the packet loss, (11) can be written as follows:

$$
\mathbf{y}_{k}^{i}=\boldsymbol{\alpha}_{k}^{i} \mathbf{C}^{i} \mathbf{x}_{k}+\boldsymbol{\alpha}_{k}^{i} \mathbf{w}_{k}^{i},
$$

where $\mathbf{y}_{k}^{i}$ is the received measurements under the condition of packet losses, and $\boldsymbol{\alpha}_{k}^{i}=\operatorname{diag}\left[\alpha_{k}^{i}(1), \ldots, \alpha_{k}^{i}\left(r^{i}\right)\right], r^{i}$ is

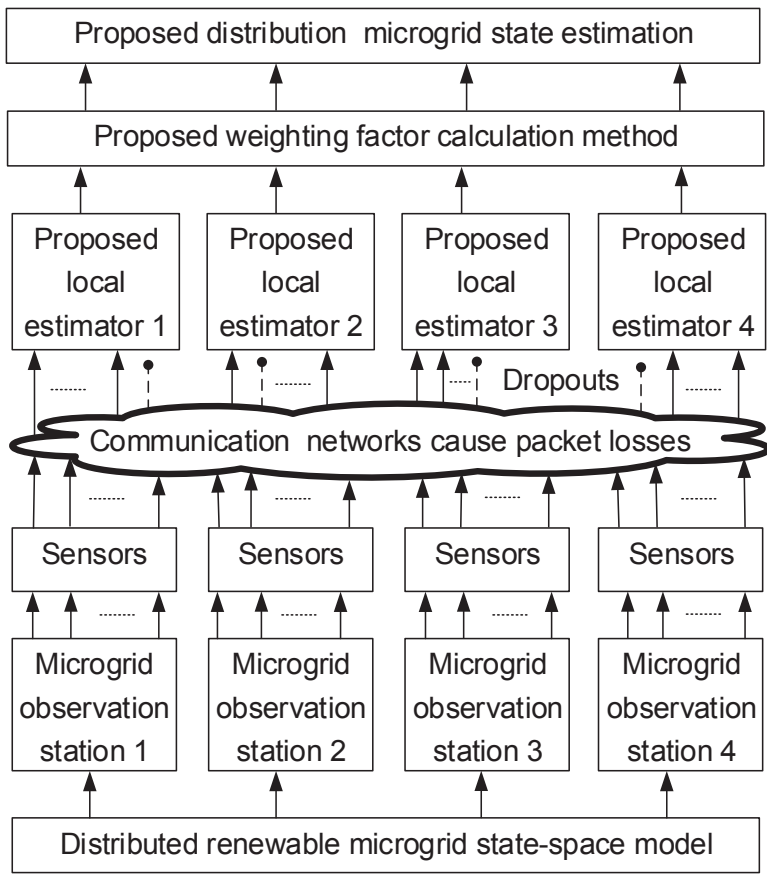

Fig. 4: Proposed distributed microgrid state estimation including packet losses.

the dimension of $\mathbf{y}_{k}^{i}$ and $\alpha_{k}^{i}(.) \in\{0,1\}$ is the Bernoulli distribution which is considered as a packet loss model in this work [9]. The packet loss can be modelled as follows:

$$
\alpha_{k}^{i}(j)= \begin{cases}1, & \text { with probability of } \lambda^{i}(j), \\ 0, & \text { with probability of } 1-\lambda^{i}(j),\end{cases}
$$

where $\lambda^{i}(j)$ is the packet arrival rate reaching at the estimator.

\section{PROBLEM FORMULATION}

Considering the packet losses, the local KF for each estimator is written as follows [18]:

$$
\begin{aligned}
& \hat{\mathbf{x}}_{k \mid k-1}^{i}=\mathbf{A}_{d} \hat{\mathbf{x}}_{k-1 \mid k-1}^{i}+\mathbf{B}_{d} \mathbf{u}_{k}, \\
& \mathbf{P}_{k \mid k-1}^{i}=\mathbf{A}_{d} \mathbf{P}_{k-1 \mid k-1}^{i} \mathbf{A}_{d}^{\prime}+\mathbf{Q}_{k}^{i}, \\
& \hat{\mathbf{x}}_{k \mid k}^{i}=\hat{\mathbf{x}}_{k \mid k-1}^{i}+\mathbf{K}_{k}^{i}\left[\mathbf{y}_{k}^{i}-\boldsymbol{\alpha}_{k}^{i} \mathbf{C}^{i} \hat{\mathbf{x}}_{k \mid k-1}^{i}\right],
\end{aligned}
$$

where $\hat{\mathbf{x}}_{k-1 \mid k-1}^{i}$ is the estimated state of the previous step, $\mathbf{P}_{k-1 \mid k-1}^{i}$ is the forecasted covariance matrix of the earlier phrase, $\hat{\mathbf{x}}_{k \mid k}^{i}$ is the local estimation and $\mathbf{K}_{k}^{i}$ is the Kalman gain. After local estimation at each estimator, the proposed distributed smart grid state estimation is described by the following expression:

$$
\hat{\mathbf{x}}_{k \mid k}^{g}=\sum_{l=1}^{N} \mathbf{W}_{k}^{l} \hat{\mathbf{x}}_{k \mid k}^{l},
$$

where $\mathbf{W}_{k}^{l}$ is the weighting matrix at the 1-th estimator at the time instant $\mathrm{k}$ and $\hat{\mathbf{x}}_{k \mid k}^{g}$ is the global estimated system state. To do that estimation, the first problem is to find an optimal Kalman gain, so that the mean squared error $E\left[\left\{\mathbf{x}_{k}-\hat{\mathbf{x}}_{k \mid k}\right\}\left\{\mathbf{x}_{k}-\hat{\mathbf{x}}_{k \mid k}\right\}^{\prime}\right]$ is minimized. The second problem 
is to find a set of optimal weighting sequence, so the local estimated covariance matrix is minimized.

\section{Proposed Methodology}

Let $\mathbf{e}^{i}$ denotes the estimation error between actual system state and estimated state of i-th estimator. Arithmetically, it can be expressed as follows:

$$
\begin{aligned}
& \mathbf{e}_{k \mid k-1}^{i}=\mathbf{x}_{k}-\hat{\mathbf{x}}_{k \mid k-1}^{i} . \\
& \mathbf{e}_{k \mid k}^{i}=\mathbf{x}_{k}-\hat{\mathbf{x}}_{k \mid k}^{i} .
\end{aligned}
$$

Now substituting (15) into (18), one can obtain the following expression:

$$
\begin{aligned}
\mathbf{e}_{k \mid k}^{i} & =\mathbf{x}_{k}-\hat{\mathbf{x}}_{k \mid k-1}^{i}-\mathbf{K}_{k}^{i}\left[\mathbf{y}_{k}^{i}-\boldsymbol{\alpha}_{k}^{i} \mathbf{C}^{i} \hat{\mathbf{x}}_{k \mid k-1}^{i}\right] \\
& =\mathbf{x}_{k}-\hat{\mathbf{x}}_{k \mid k-1}^{i}-\mathbf{K}_{k}^{i}\left[\boldsymbol{\alpha}_{k}^{i} \mathbf{C}^{i} \mathbf{x}_{k}+\boldsymbol{\alpha}_{k}^{i} \mathbf{w}_{k}^{i}-\boldsymbol{\alpha}_{k}^{i} \mathbf{C}^{i} \hat{\mathbf{x}}_{k \mid k-1}^{i}\right] \\
& =\left[\mathbf{I}-\boldsymbol{\alpha}_{k}^{i} \mathbf{K}_{k}^{i} \mathbf{C}^{i}\right]\left[\mathbf{x}_{k}-\hat{\mathbf{x}}_{k \mid k-1}^{i}\right]-\boldsymbol{\alpha}_{k}^{i} \mathbf{K}_{k}^{i} \mathbf{w}_{k}^{i} \\
& =\left[\mathbf{I}-\boldsymbol{\alpha}_{k}^{i} \mathbf{K}_{k}^{i} \mathbf{C}^{i}\right] \mathbf{e}_{k \mid k-1}^{i}-\boldsymbol{\alpha}_{k}^{i} \mathbf{K}_{k}^{i} \mathbf{w}_{k}^{i} .
\end{aligned}
$$

Now, the estimation error covariance matrix is defined by:

$$
\mathbf{P}_{k \mid k}^{i}=E\left[\mathbf{e}_{k \mid k}^{i} \mathbf{e}_{k \mid k}^{i}\right] .
$$

By substituting (19) into (20), one can obtain the following estimation error covariance matrix:

$$
\begin{gathered}
\mathbf{P}_{k \mid k}^{i}=\lambda_{k}^{i}\left[\mathbf{I}-\mathbf{K}_{k}^{i} \mathbf{C}^{i}\right] \mathbf{P}_{k \mid k-1}^{i}\left[\mathbf{I}-\mathbf{K}_{k}^{i} \mathbf{C}^{i}\right]^{\prime}+\lambda_{k}^{i} \mathbf{K}_{k}^{i} \mathbf{R}_{k}^{i} \mathbf{K}_{k}^{\prime}{ }^{i}+ \\
\left(1-\lambda_{k}^{i}\right) \mathbf{P}_{k \mid k-1}^{i} .
\end{gathered}
$$

Taking the partial derivative of (21) with respect to $\mathbf{K}_{k}^{i}$ yields:

$$
\begin{gathered}
\frac{\partial\left[\operatorname{tr} \mathbf{P}_{k \mid k}^{i}\right]}{\partial \mathbf{K}_{k}^{i}}=-2 \lambda_{k}^{i} \mathbf{P}_{k \mid k-1}^{i} \mathbf{C}^{\prime i}+2 \lambda_{k}^{i} \mathbf{K}_{k}^{i} \mathbf{C}^{i} \mathbf{P}_{k \mid k-1}^{i} \mathbf{C}^{\prime}+ \\
2 \lambda_{k}^{i} \mathbf{K}_{k}^{i} \mathbf{R}_{k}^{i}
\end{gathered}
$$

Setting $\frac{\partial\left[\operatorname{tr} \mathbf{P}_{k \mid k}^{i}\right]}{\partial \mathbf{K}_{k}^{i}}=\mathbf{0}$ and the Kalman gain is given by:

$$
\mathbf{K}_{k}^{i}=\mathbf{P}_{k \mid k-1}^{i} \mathbf{C}^{\prime}{ }^{i}\left[\mathbf{C}^{i} \mathbf{P}_{k \mid k-1}^{i} \mathbf{C}^{\prime i}+\mathbf{R}_{k}^{i}\right]^{-1} .
$$

The proposed distributed state estimation (16) is numerically described by the following expression:

$$
\hat{\mathbf{x}}_{k \mid k}^{g}=\mathbf{W}_{k}^{1} \hat{\mathbf{x}}_{k \mid k}^{1}+\mathbf{W}_{k}^{2} \hat{\mathbf{x}}_{k \mid k}^{2}+\mathbf{W}_{k}^{3} \hat{\mathbf{x}}_{k \mid k}^{3}+\mathbf{W}_{k}^{4} \hat{\mathbf{x}}_{k \mid k}^{4} .
$$

In order to find the optimal weighting factors, one can search for the weighting factors that elucidates the following minimization problem:

$$
\begin{array}{ll}
\operatorname{minimise} & \operatorname{trace}[\mathbf{S}] \\
\text { subject to } \quad & \mathbf{W}_{k}^{1} \hat{\mathbf{P}}_{k \mid k}^{1} \mathbf{W}_{k}^{\prime 1}+\mathbf{W}_{k}^{2} \hat{\mathbf{P}}_{k \mid k}^{2} \mathbf{W}_{k}^{\prime 2}+\mathbf{W}_{k}^{3} \hat{\mathbf{P}}_{k \mid k}^{3} \mathbf{W}_{k}^{\prime 3}+ \\
& \mathbf{W}_{k}^{4} \hat{\mathbf{P}}_{k \mid k}^{4} \mathbf{W}_{k}^{\prime 4} \leq \mathbf{S} \\
& \mathbf{W}_{k}^{1}+\mathbf{W}_{k}^{2}+\mathbf{W}_{k}^{3}+\mathbf{W}_{k}^{4}=\mathbf{I}_{4}
\end{array}
$$

where $\hat{\mathbf{P}}_{k \mid k}^{i}$ is the $\mathrm{i}$-th estimation error covariance matrix by (21), and $\mathbf{S}$ is the auxiliary variable for minimizing the global estimator error covariance. Consequently, the error covariance

\begin{tabular}{|c|c|c|c|c|c|}
\hline Symbols & Values & Symbols & Values & Symbols & Values \\
\hline$f_{p r}$ & 0.4 & $V_{r}$ & $750 \mathrm{~V}$ & $\overline{\mathbf{Q}^{i}}$ & $0.00001 * \mathbf{I}$ \\
\hline$P_{p P}$ & 1 & $R_{p}$ & $0.01 \Omega$ & $\mathbf{R}^{4}$ & $0.000017 * \mathbf{I}$ \\
\hline$E_{p}$ & $300 \mathrm{~V}$ & $L_{p}$ & $0.01 \mathrm{H}$ & $\mathbf{R}^{3}$ & $0.000015^{*} \mathbf{I}$ \\
\hline$f_{b r}$ & 0.67 & $V_{b r}$ & $500 \mathrm{~V}$ & $\mathbf{R}^{2}$ & $0.000012 * \mathbf{I}$ \\
\hline$L_{b}$ & $0.01 \mathrm{H}$ & $C$ & $0.01 \mathrm{~F}$ & $\mathbf{R}^{1}$ & $0.00001 * \mathbf{I}$ \\
\hline$P_{\text {load }}$ & $300 \mathrm{Kw}$ & V & $800 \mathrm{~V}$ & $\lambda^{4}$ & 0.97 \\
\hline$R_{\text {load }}$ & $500 \Omega$ & $i_{p r}$ & $350 \mathrm{~A}$ & $\lambda^{3}$ & 0.91 \\
\hline$P_{p I}$ & 1 & $R_{b}$ & $0.01 \Omega$ & $\lambda^{2}$ & 0.94 \\
\hline$i_{b r}$ & $50 \mathrm{~A}$ & $C_{b}$ & $1000 \mathrm{~F}$ & $\lambda^{1}$ & 0.97 \\
\hline$P_{b P}$ & 1 & $P_{b I}$ & 1 & $\Delta t$ & 0.00001 \\
\hline
\end{tabular}
is minimized, so that the estimated states match the true states. By using the semidefinite programming variable $\mathbf{S}$, the distribution estimation problem is converted to the convex problem which can be solved effectively and efficiently. Now according to the standard Schur's complement, (26) can be transformed into the following linear matrix inequality form:

$$
\left[\begin{array}{ccccc}
-\mathbf{S} & \mathbf{W}_{k}^{1} & \mathbf{W}_{k}^{2} & \mathbf{W}_{k}^{3} & \mathbf{W}_{k}^{4} \\
\mathbf{W}_{k}^{\prime 1} & -\left(\hat{\mathbf{P}}_{k \mid k}^{1}\right)^{-1} & \mathbf{0} & \mathbf{0} & \mathbf{0} \\
\mathbf{W}_{k}^{\prime 2} & \mathbf{0} & -\left(\hat{\mathbf{P}}_{k \mid k}^{2}\right)^{-1} & \mathbf{0} & \mathbf{0} \\
\mathbf{W}_{k}^{\prime 3} & \mathbf{0} & \mathbf{0} & -\left(\hat{\mathbf{P}}_{k \mid k}^{3}\right)^{-1} & \mathbf{0} \\
\mathbf{W}_{k}^{\prime 4} & \mathbf{0} & \mathbf{0} & \mathbf{0} & -\left(\hat{\mathbf{P}}_{k \mid k}^{4}\right)^{-1}
\end{array}\right] \leq \mathbf{0} \text {. }
$$

Finally, one can formulate the optimization problem as follows:

$$
\begin{array}{ll}
\text { minimise } & \text { trace }[\mathbf{S}] \\
\text { subject to } & \text { Hold }(27), \text { and Hold }(28) .
\end{array}
$$

\section{Simulation RESUlTS}

The system parameters used in this simulation are summarized in Table I. As this is an open-loop system without control,

TABLE I: Parameters of system configuration for adaptive-then-combine case.

the system dynamics cannot be guaranteed to be stable. That is as can be seen in the simulation results, the state will fluctuate which is determined by the open-loop system A matrix. The simulation results demonstrate that the proposed approach is able to estimate the microgrid states with high estimation accuracy. For instance, from Fig. 6 it is observed that the proposed method is able to estimate the microgrid state within 4 steps $^{1}$. This is due to the fact that the proposed Kalman gain is able to minimise the packet losses and noise effect on the estimation process. Secondly, the proposed weighting factor calculation method is able to find the optimal weighting matrices, so that the estimation error can be minimized. Note that $f_{p I}$ is not constant, which can be seen if we increase the simulation time.

\section{CONCLUSION}

From the perspective of the environmental, technical, economic and social benefits from the microgrid at the community level, we have investigated the problem of distributed state estimation with packet dropouts in smart grids. After modelling the renewable microgrid in a distributed way, this paper proposes an adaptive algorithm which can minimize the estimation error covariance matrix of the local estimators. The proposed novel optimization method reduces the estimation

\footnotetext{
${ }^{1}$ Other states have similar estimation performance.
} 


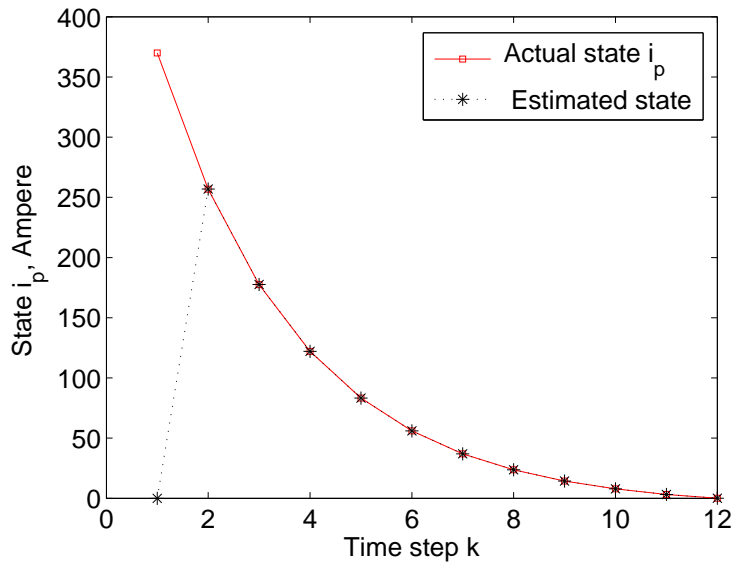

Fig. 5: Real values and estimated values of $i_{p}$.

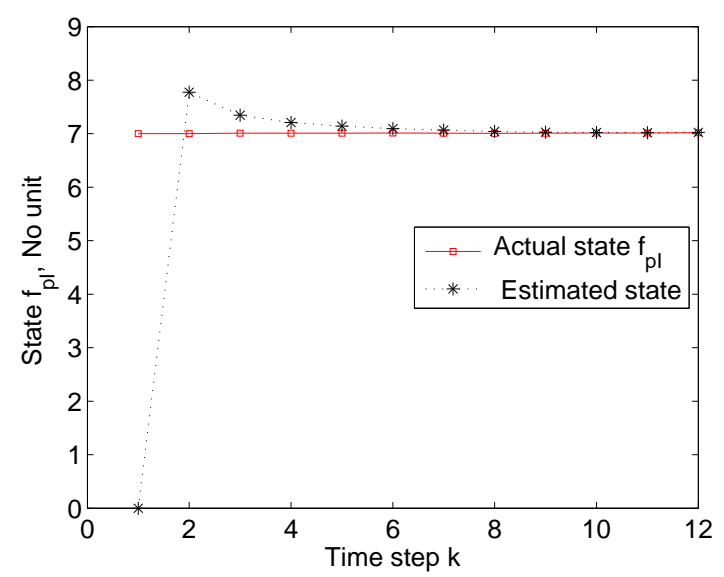

Fig. 6: Real values and estimated values of $f_{p I}$.

error so that the estimated state can well track the true system state. Simulation results indicate that the proposed distributed approach is able to properly monitor the microgrid. The delay and packet losses between the microgrid and state estimator will be considered in the future work.

\section{REFERENCES}

[1] G. Mendes, J. Von Appen, and C. Ioakimidis, "Integrated energy microgrids for community-scale systems: Case study research in the azores islands," in Proc of the International Conference of the European Energy Market, 2011, pp. 382-387.

[2] X. Yu, Z. Jiang, and Y. Zhang, "Control of parallel inverter-interfaced distributed energy resources," in Proc of the International Conference on Energy 2030, 2008, pp. 1-8.

[3] T. Orhan, G. Shafiullah, A. Stojcevski, and A. Oo, "A feasibility study on microgrid for various islands in Australia," in Proc of the International Conference Power Engineering Conference, 2014, pp. 18.

[4] R. M. Kamel, A. Chaouachi, and K. Nagasaka, "Three control strategies to improve the microgrid transient dynamic response during isolated mode: A comparative study," IEEE Transactions on Industrial Electronics, vol. 60, no. 4, pp. 1314-1322, 2013.

[5] Y. V. Makarov, P. Du, M. C. Kintner-Meyer, C. Jin, and H. F. Illian, "Sizing energy storage to accommodate high penetration of variable

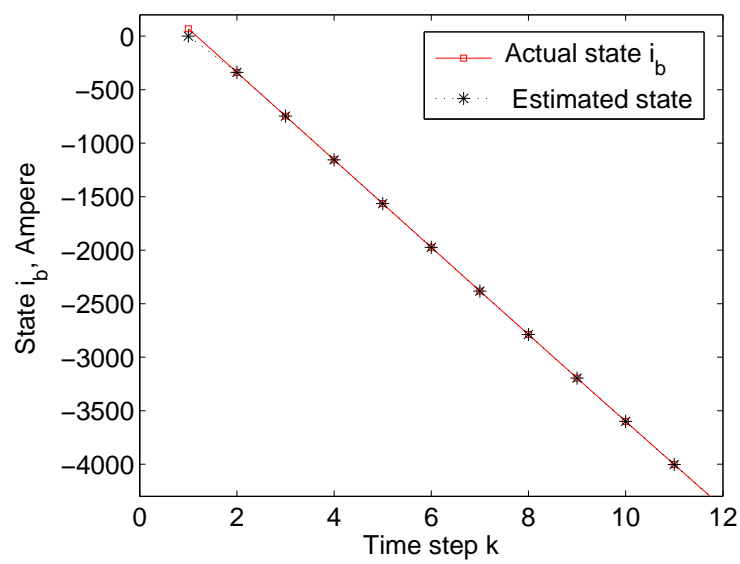

Fig. 7: Real values and estimated values of $i_{b}$.

energy resources," IEEE Transactions on Sustainable Energy, vol. 3, no. 1, pp. 34-40, 2012.

[6] A. Arefi, G. Ledwich, and B. Behi, "An efficient DSE using conditional multivariate complex Gaussian distribution," IEEE Transactions on Smart Grid, vol. 6, no. 4, pp. 2147-2156, 2015.

[7] A. K. Singh and B. C. Pal, "Decentralized dynamic state estimation in power systems using unscented transformation," IEEE Transactions on Power Systems, vol. 29, no. 2, pp. 794-804, 2014.

[8] H. Dong, Z. Wang, J. Lam, and H. Gao, "Distributed filtering in sensor networks with randomly occurring saturations and successive packet dropouts," International Journal of Robust and Nonlinear Control, vol. 24, no. 12, pp. 1743-1759, 2014.

[9] B. Sinopoli, L. Schenato, M. Franceschetti, K. Poolla, M. Jordan, and S. Sastry, "Kalman filtering with intermittent observations," IEEE Transactions on Automatic Control, vol. 49, no. 9, pp. 1453-1464, 2004.

[10] D. Han, Y. Mo, J. Wu, S. Weerakkody, B. Sinopoli, and L. Shi, "Stochastic event-triggered sensor schedule for remote state estimation," IEEE Transactions on Automatic Control, vol. 60, no. 10, pp. 26612675, 2015.

[11] T. Sui, K. You, M. Fu, and D. Marelli, "Stability of MMSE state estimators over lossy networks using linear coding," Automatica, vol. 51, pp. 167-174, 2015.

[12] A. K. Singh, R. Singh, and B. C. Pal, "Stability analysis of networked control in smart grids," IEEE Transactions on Smart Grid, vol. 6, no. 1, pp. 381-390, 2015.

[13] M. M. Rana, L. Li, and S. Su, "Distributed state estimation using RSC coded smart grid communications," IEEE Access Journal, vol. 3, no. 1, pp. 1-10, 2015.

[14] G. Rigatos, P. Siano, and N. Zervos, "A distributed state estimation approach to condition monitoring of nonlinear electric power systems," Asian Journal of Control, vol. 15, no. 3, pp. 849-860, 2013.

[15] F. Zhao, N. Li, Z. Yin, and X. Tang, "Small-signal modeling and stability analysis of DC microgrid with multiple type of loads," in Proc of the International Conference on Power System Technology, 2014, pp. 3309-3315.

[16] M. Farhadi and O. Mohammed, "Adaptive energy management in redundant hybrid DC microgrid for pulse load mitigation," IEEE Transactions on Smart Grid, vol. 6, no. 1, pp. 54-62, 2015.

[17] S. Chowdhury, G. Taylor, S. Chowdhury, A. Saha, and Y. Song, "Modelling, simulation and performance analysis of a PV array in an embedded environment," in Proc of the Universities Power Engineering Conference, 2007, pp. 781-785.

[18] F. S. Cattivelli and A. H. Sayed, "Diffusion LMS strategies for distributed estimation," IEEE Transactions on Signal Processing, vol. 58, no. 3, pp. 1035-1048, 2010. 